\title{
Functionalized graphene foam as electrode for improved electrochemical storage
}

\author{
A. Bello ${ }^{1}$, M. Fabiane ${ }^{1}$, D. Y. Momodu ${ }^{1}$, S. Khamlich ${ }^{1}$, J. K. Dangbegnon ${ }^{1}$ and N. Manyala $^{1 *}$ \\ ${ }^{1}$ Department of Physics, Institute of Applied Materials, SARChI Chair in Carbon Technology and Materials, University of Pretoria, Pretoria \\ 0028, South Africa \\ Corresponding author: N Manyala. \\ E-mail address: ncholu.manyala@up.ac.za
}

Tel: +27(0)12420 3549, Fax: +27(0)12420 2516

\begin{abstract}
We report on a non-covalent functionalization of graphene foam (GF) synthesised via chemical vapor deposition (CVD). The GF was treated with pyrene carboxylic acid (PCA) which acted as a source of oxygen and/ or hydroxyl groups attached to the surface of the graphene foam for its electrochemical performance improvement. The modified graphene surface enabled a high pseudocapacitive effect on the GF. A specific capacitance of 133.3 $\mathrm{F} \mathrm{g} \mathrm{g}^{-1}$, power density $\sim 145.3 \mathrm{~kW} \mathrm{~kg}^{-1}$, and energy density $\sim 4.7 \mathrm{~W} \mathrm{~h} \mathrm{~kg}^{-1}$ was achieved based on the functionalized foam in $6 \mathrm{M} \mathrm{KOH}$ aqueous electrolyte. The results suggest that non-covalent functionalization might be an effective approach to overcome the restacking problem associated with graphene electrodes and also signifies the importance of surface functionalities in graphene based electrode materials.
\end{abstract}

Keywords: Graphene foam (GF), pyrene carboxylic acid (PCA), chemical vapor deposition CVD, and supercapacitor.

\section{Introduction}

Over the past few years, the interesting and fascinating properties of graphene have attracted a lot of research activities from various fields for both applied and fundamental studies [1]. This material is a two-dimensional (2D) allotrope of carbon comprising of a monoatomic sheet of $\mathrm{sp}^{2}$ hybridized carbon atoms tightly bound in a honeycomb lattice that exhibits unique electronic structure, high specific surface area $\left(2630 \mathrm{~m}^{2} \mathrm{~g}^{-1}\right)$, high electrical conductivity, chemical stability and structural flexibility [2-4]. These properties have generated a lot of curiosities for researchers to explore graphene and graphene based functional materials for a wide range of 
possible applications such as electronics, sensors, composite materials, photocatalysis, energy storage and conversion [5-10]. Supercapacitors are energy storage systems that have attracted interest because of their unique mechanism of storing energy by accumulating charges at the electrode-electrolyte interface [11]. They are power devices with versatile range of applications such as portable electronic devices, hybrid electric device and high-pulse power backup [12]. Due to the long cycle life and rapid charge-discharge, supercapacitors could be used as hybrid systems in batteries and fuel cells [13]. However, such excellent devices require electrode materials with high specific surface area, excellent electrical conductivity and porosity. Carbonaceous material such as activated carbon $[14,15]$, CNTs [16] and more recently graphene [17] are considered as possible materials for energy storage because of their conductivity and high surface to volume ratio. Therefore several research activities have been focused on modification of these materials for improved energy storage by increasing the contact area at the electrode-electrolyte interface.

Mechanical exfoliation of graphite is known to produce graphene flakes with exceptional electronic properties. However, this method of graphene production is suitable for fundamental studies and is unsuitable for large scale production and practical applications [4]. To date, a great number of works have reported large scale production of graphene based on chemical modification of Hummer's method $[18,19]$. This technique involves the oxidation of graphite to graphene oxide using strong acids and oxidizing agent, and subsequently using strong reducing agent such as hydrazine $\left(\mathrm{N}_{2} \mathrm{H}_{4}\right)$ and sodium borohydride $\left(\mathrm{NaBH}_{4}\right)$ for reduction of the graphene oxide to graphene. The graphene sheets obtained from this method are usually defective due to the oxidation or reduction steps, and hence restacking or irreversible aggregation of the sheets often lead to decrease in the electrical conductivity during the reduction process and hence exhibit low electrochemical performance [20, 21]. The graphene sheets tend to aggregate forming graphite insolubility in most common solvent due to strong van der Waals interactions [22]. To overcome this problem, fillers, spacers or pseudocapacitive materials are considered to prevent restacking of the graphene sheet thereby improving the performance of the capacitors. Alternatively, functionalization of the graphene sheets with carbonyl or hydroxyl groups could also reduce the degree of aggregation of the sheets as well as creating pathways for ion transportation [23]. A variety of surface functionalization of graphene have been reported, some of which include; functionalization with aromatic molecules [24], amidiation [25], polymers and esterification of carboxylic groups in poly(vinyl alcohol) PVA [26, 27], salinization with hydroxyl groups [28] and composite reinforcement [29]. However, most of these functionalization techniques distort the $\mathrm{sp}^{2}$ structure of graphene and tend to convert it into $\mathrm{sp}^{3}$, leading to 
decrease in charge mobility and inferior electronic properties [30]. To overcome this problem, non-covalent functionalization has been employed because of its ability to manipulate the electronic and chemical properties without disrupting the $\mathrm{sp}^{2}$ hybrid structure of graphene. Non-covalent functionalization is particularly attractive because of the possibility of adsorbing various functional groups onto the graphene surface through the weak van der Waals (vdW) interaction or the common non-destructive $\pi-\pi$ interaction mechanism thereby preserving superior physical and electronic properties of graphene [31].

To date, a number of techniques for non-covalent functionalization of graphene for desirable applications have been investigated. For example, non-covalent molecular functionalization has been used to dope single layer graphene and to induce band-gap in bilayer graphene $[32,33]$, for surface transfer doping of diamond (100) with tetrafluorotetracyanoquinodimethane [34], for reduction of sheet resistance in organic photovoltaic cells (OPVs) [35], to improve the selectivity and sensitivity of molecular biosensors [36, 37] and for electrochemical storage applications [38, 39]. Therefore, a step towards developing high performance electrode material for electrochemical capacitors based on functionalized graphene sheet is highly desirable. The efficient adsorption of these functional groups onto the graphene sheet is essential for their application and development.

In this work we report on the growth of highly crystalline three dimensional graphene foam using nickel foam as a template via chemical vapour deposition (CVD) and modification of the sheets by employing non-covalent functionalization technique [40]. The successful functionalization has been achieved by a simple non-covalent functionalization with 1-pyrenecarboxylic acid (PCA) in N, N dimethyformamide (DMF). Due to the amphilic nature of PCA with high solubility in DMF, an effective complex is formed which leads to attachment of PCA molecules on the surface of the graphene. The as-functionalized material was used for fabrication of electrode for supercapacitor device. The device demonstrated good electrochemical performance including excellent cycle stability in a two electrode symmetric configuration.

\section{Experimental}

\section{Synthesis of graphene foam (GF).}

Graphene foam (GF) was synthesised by CVD onto a catalytic nickel foam (Munich Germany). The detailed procedure is described in our previous report [41]. To provide mechanical support for the GF during etching of the nickel, polymethylmethacrylate (PMMA) was drop coated on the sample and baked at $180{ }^{\circ} \mathrm{C}$ for 30 minutes. The samples were then placed in $3 \mathrm{M} \mathrm{HCl}$ solution at $80{ }^{\circ} \mathrm{C}$, left overnight to ensure complete removal 
of the nickel. The resulting GF sample was placed in acetone at $50{ }^{\circ} \mathrm{C}$ for 30 minutes to remove the PMMA. The samples were then rinsed with deionised water and dried.

\section{Thermal Treatment (Oxidation) of Graphene Foam in Air.}

The GF was transferred into a crucible boat and placed inside an open air furnace and heated at $550{ }^{\circ} \mathrm{C}$ for $1 \mathrm{~h}$ for oxidation of the sample.

\section{Functionalization of graphene foam.}

The oxidised GF was functionalized via a non-covalent method using 1-pyerene carboxylic acid (PCA) and dimethylformamide (DMF). $0.5 \mathrm{~g}$ of PCA was dissolved in $50 \mathrm{ml}$ of DMF solution. $20 \mathrm{mg}$ of oxidised GF was then added to the mixture, ultrasonicated at $90{ }^{\circ} \mathrm{C}$ for 12 hours to obtain a homogeneous dispersed mixture. The functionalized graphene was separated by centrifugation at $5000 \mathrm{rpm}$ and the supernatant solution was collected in a vial. The solid powder was washed with DMF several times and dried at $60{ }^{\circ} \mathrm{C}$ in an electric oven. Both, solution and solid powder will be analyzed in this work.

\section{Characterization and measurements.}

X-ray diffraction (XRD) patterns of the samples were collected using an XPERT-PRO diffractometer (PANalytical BV, Netherlands) with theta/2theta geometry, operating with a cobalt tube at $35 \mathrm{kV}$ and $50 \mathrm{~mA}$. The concentration of the materials was determined by UV-vis spectra measurements using a Perkin Elmer Lambda $40 \mathrm{UV}$-Vis-NIR spectrophotometer. All measurements were performed in air in the 250-500 $\mathrm{nm}$ range with a resolution of $1 \mathrm{~nm}$. Raman spectra were recorded using a WiTec-alpha 300R+ confocal Raman spectrometer (WiTec $\mathrm{GmbH})$. The excitation source was the 532-nm laser line focused through a numerical aperture of 0.9 and 100x magnification. The surface morphology of the sample was investigated using the high resolution Zeiss Ultra plus 55 field emission scanning electron microscope (FESEM) operated at $2.0 \mathrm{kV}$. Transmission electron microscopy (TEM) images were obtained with a JEOL JEM-2100F microscope operated at $200 \mathrm{kV}$. XPS measurements were obtained using a VersaProbe 5000 with an Al Ka X-ray source.

The electrochemical measurements were carried out in a conventional two electrode configuration. The working electrodes were prepared by mixing 85 wt. \% of GF with 10 wt. $\%$ carbon black and 5 wt. $\%$ polyvinylidene difluoride (PVdF) binder in an agate mortar. The mixture was then dissolved in 1-methyl-2-pyrrolidinone (NMP) to form a paste. The homogenous paste was coated onto a Ni foam current collector and dried at $60{ }^{\circ} \mathrm{C}$ in an oven for 8 hours to ensure complete evaporation of the NMP. The total mass of the active material in the 
working electrodes was $\sim 4 \mathrm{mg}$, and the two electrodes with identical mass were selected for measurement. The coin cell was made by sandwiching both electrodes between a filter paper which acts as the separator. The cell was evaluated in a $6 \mathrm{M} \mathrm{KOH}$ aqueous electrolyte solution using cyclic voltammetry $(\mathrm{CV})$, galvanostatic chargedischarge (GV) and electrochemical impedance spectroscopy (EIS) using a Bio-logic SP-300 PGSTAT. The EIS plot was evaluated in the frequency region of $100 \mathrm{kHz}$ to $10 \mathrm{~m} \mathrm{~Hz}$.
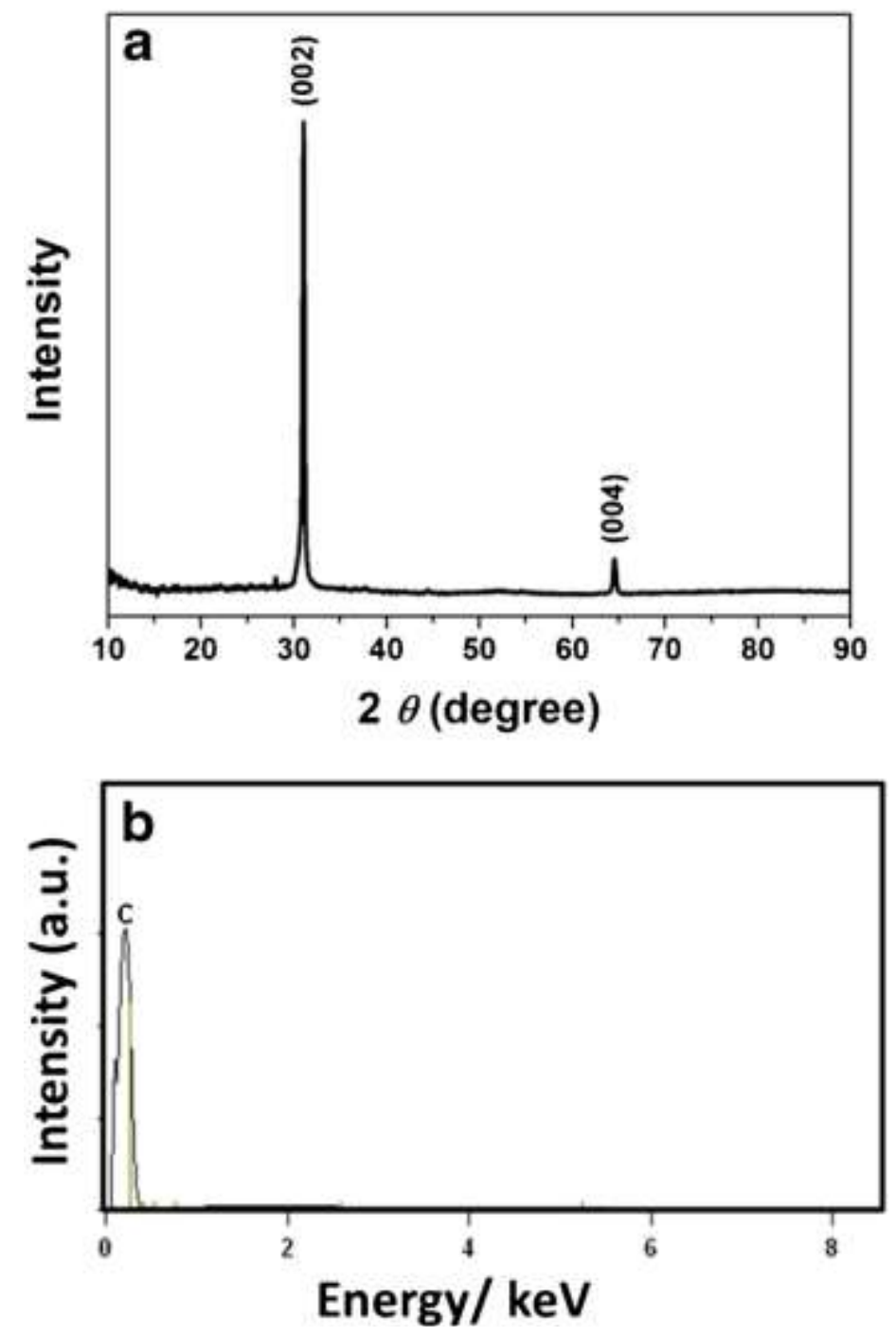

Figure 1 XRD and EDX pattern of GF after etching process.

\section{Results and discussion}

XRD pattern and EDX of the GF is shown in figure 1. Figure 1(a) shows diffraction peaks at $2 \theta=31^{\circ}$ and $64.5^{\circ}$ which corresponds to the (002) and (004) reflections of hexagonal graphite, respectively. No diffraction peaks corresponding to nickel is detected which is a clear indication that nickel has been completely removed from the 
graphene foam during the etching process. EDX elemental analysis was also used to confirm the removal of nickel as shown in figure 1 (b) proving only the presence of carbon in the sample.

The formation of functionalized GF is confirmed by UV-vis absorption spectroscopy. PCA is a polyaromatic hydrocarbon that contains a polar carboxylic acid group and a nonpolar pyrene group. The polar group makes it soluble in most common solvent such as DMF and methanol which also have polar groups attached to them. The pyrene group consists of a $\pi$-conjugated network structure surrounded by clouds of delocalized $\pi$ electrons. The pyrene group has a mixed hexagonal and aromatic structure thereby forming a $\pi-\pi$ aromatic interaction. The resulting PCA-GF complex is soluble in DMF due to the presence of the polar group attached to the surface of the GF. Figure 2 shows the UV-vis absorption of the functionalized samples before and after washing them with DMF. The change of color in the solution before and after reaction (shown in the inset to figure 2) also proves

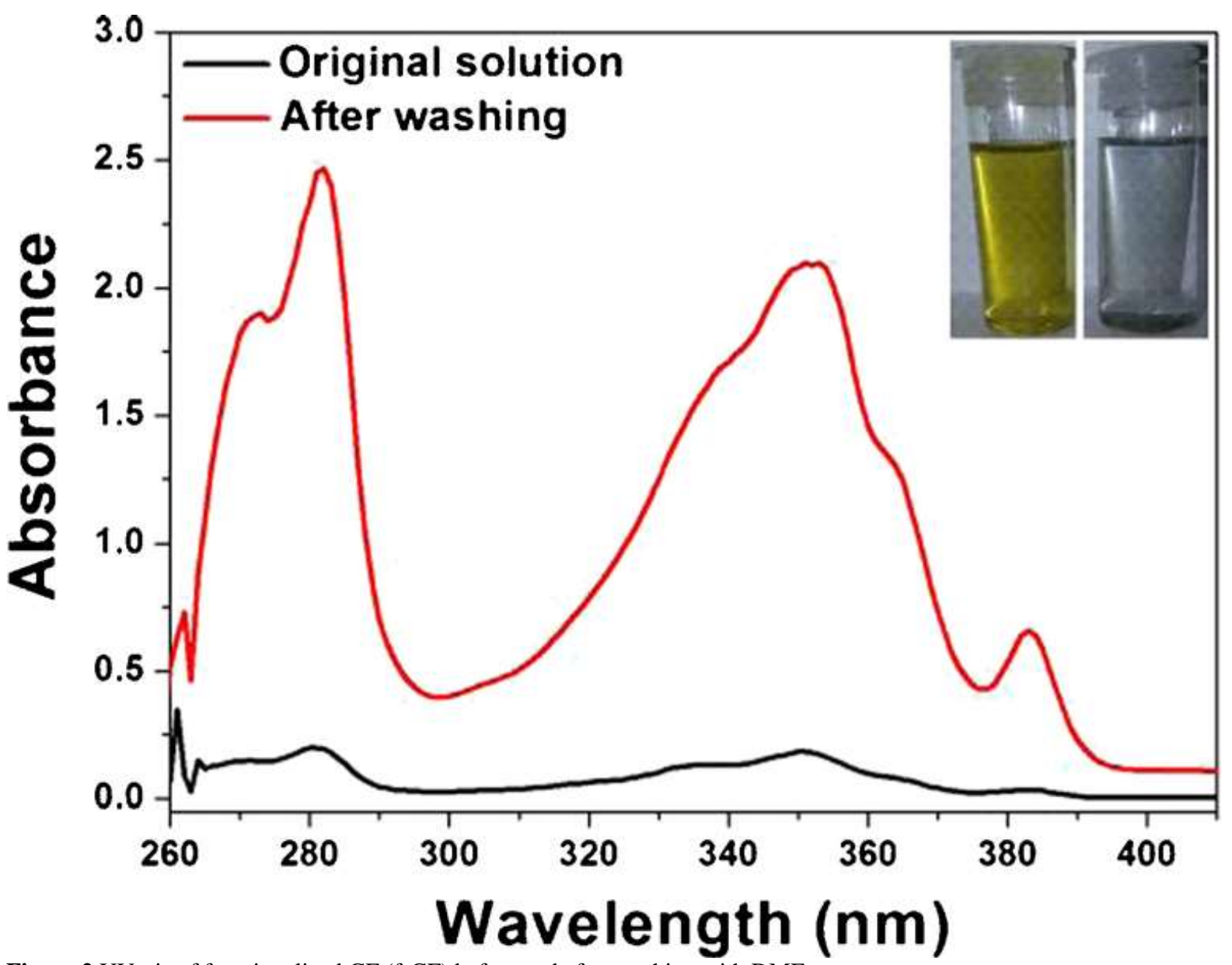

Figure 2 UV-vis of functionalized GF (f-GF) before and after washing with DMF.

the addition of PCA molecules to the GF. The aqueous solution remains very stable with very little precipitates observed after one week of storage. Such excellent dispersibility of GF makes it very favourable for further 
applications. From this figure, very weak absorbance peaks are observed for unwashed functionalized GF. This weak absorbance is due to the presence of GF and excess unreacted PCA that renders the solution opaque [39]. This unreacted PCA was removed by washing and centrifuging the solution several times. After washing, absorption peaks for PCA became clearly distinguishable evidencing PCA molecules functionalized on the GF.

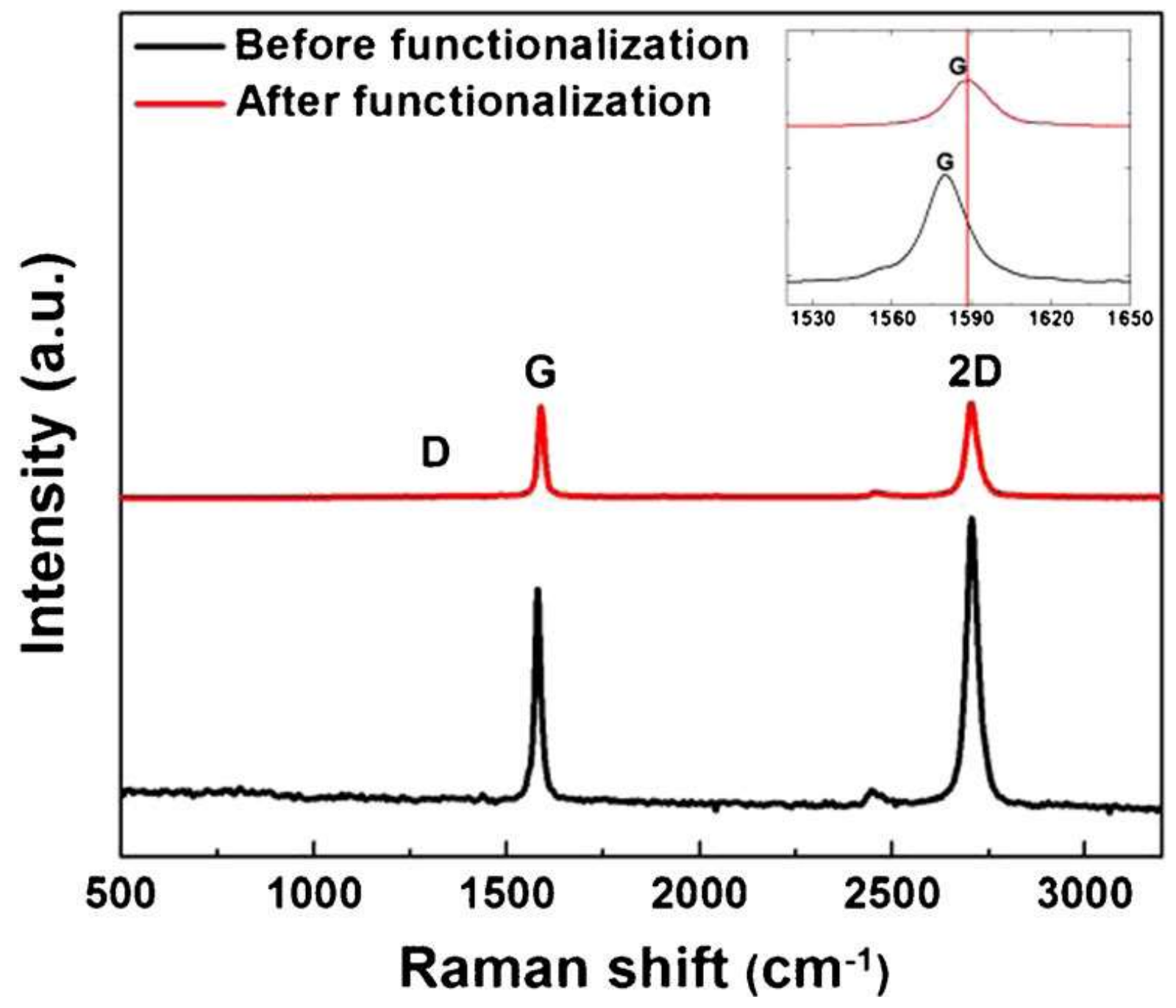

Figure 3 Raman spectra of as grown GF and functionalized GF (f-GF).

Figure 3 shows the Raman spectrum of few layer graphene flakes forming the GF. The spectrum consists of two major peaks at $\left(1579.9 \mathrm{~cm}^{-1}\right)$ and $\left(2705.7 \mathrm{~cm}^{-1}\right)$. These peaks correspond to the $\mathrm{G}$ and $2 \mathrm{D}$ modes of graphene. The $\mathrm{G}$ peak is due to the in-plane vibrations of the $\mathrm{sp}^{2}$ carbon atoms in the lattice while the $2 \mathrm{D}$ band originate from a second-order process, involving two in-plane transverse optical (iTO) phonons near the K point for the 2D band or one iTO phonon and one defect in the case of the D-band. The symmetric shape and higher intensity of the 2D peak compared to the G-peak indicates few layers graphene. The absence of the D-peak related to the 
presence of disorder at $1350 \mathrm{~cm}^{-1}$, shows that the GF is of high crystallinity [42]. The absence of the disorder peak after functionalization also indicates that the sample preserved its $\mathrm{sp}^{2}$ hybridization. The inset to figure 3 evidences a red-shift of G-peak $\left(1589.7 \mathrm{~cm}^{-1}\right)$ after functionalization. The $\mathrm{G}$ band shift and reduction of the 2D are result of charge transfer between the graphene and PCA molecules [43, 44], thus indicating surface functionalization of GF surface with PCA molecules via non-covalent method.

To further confirm the modification of the GF by PCA, X-ray photoelectron spectroscopy (XPS) was performed to know the content of oxygen and also to estimate the degree of functionalization. As shown in figure 4 the $\mathrm{C} 1 \mathrm{~s}$ spectrum of f-GF can be fitted into four peaks at 284.5, 285.1, 286.7, and $289.1 \mathrm{eV}$ corresponding to carbon atoms in four functional groups: non-oxygenated carbon $(\mathrm{C}-\mathrm{C}$ or $\mathrm{C}=\mathrm{C}, 284.5 \mathrm{eV})$, carbon in $\mathrm{C}-\mathrm{O}$ bonds $(285.1$ $\mathrm{eV})$, carbonyl carbon $(\mathrm{C}=\mathrm{O}, 286.7 \mathrm{eV})$ and carboxylate carbon $(\mathrm{O}-\mathrm{C}=\mathrm{O}, 289.1 \mathrm{eV})$ validating presence and significant amount of functional groups in the sample $[45,46]$.

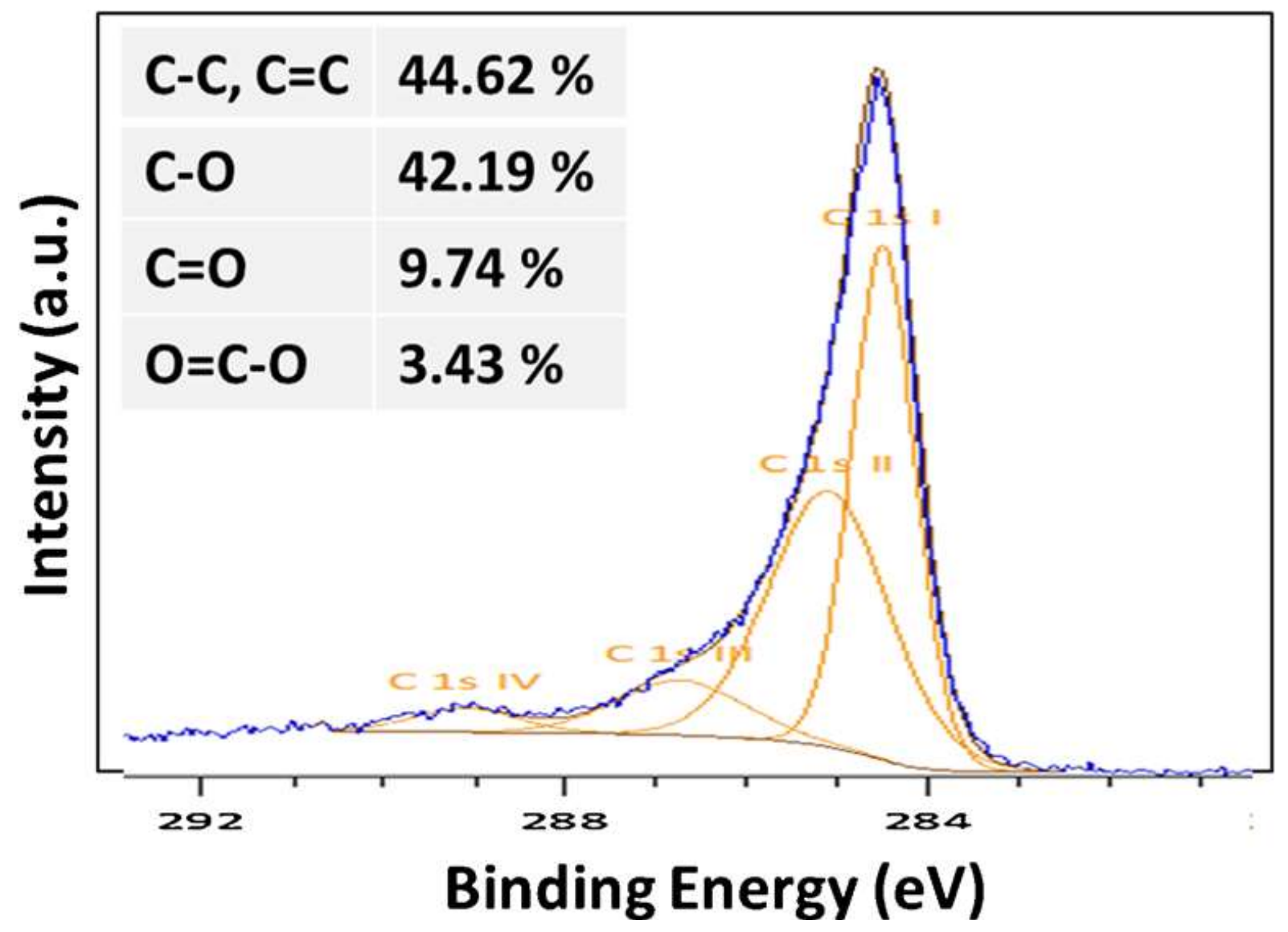

Figure $4 \mathrm{C} 1 \mathrm{~s}$ XPS profile of f-GF. 
The surface morphology of both GF and f-GF was characterized by scanning electron microscopy (SEM) and transmission electron microscopy (TEM). As shown in figure 5a, the graphene takes shape of the Ni foam template which is a porous three dimensional (3D) structure [40]. The 3D graphene structure provides abundant surface and binding sites for adsorption of hydroxyl groups. In other words this network structure of the GF provides an excellent platform for accommodating and integrating functional groups for applications due to the large inherent surface area of the GF. Inset to this figure shows high magnification image of the sheets which reveals high crystallinity. It also reveals the presence of wrinkles and ripples on the graphene sheets which is due to the different thermal expansion coefficients of $\mathrm{Ni}$ and graphene during the CVD synthesis [47]. Figure 5b shows the image of the functionalized GF. It can be observed that the 3D structure was distorted during functionalization process (sonification) and the extremely small thickness of the resulting f-GF sheets lead to a wrinkled topology [29]. Inset also shows that the sheets still maintain their morphology after functionalization. A TEM image of $\mathrm{f}-\mathrm{GF}$ is shown in figure $5 \mathrm{c}$ which shows the presence of few layers as confirmed by Raman spectroscopy. The inset shows the selected area electron diffraction (SAED) pattern that displays bright hexagonal rings corresponding to the (100) reflection from the graphene plane, suggesting the $\mathrm{sp}^{2}$ hybrid structure and electronic properties of the GF were preserved after functionalization. This is very important for preparation of electrode material based on the functionalized graphene foam.
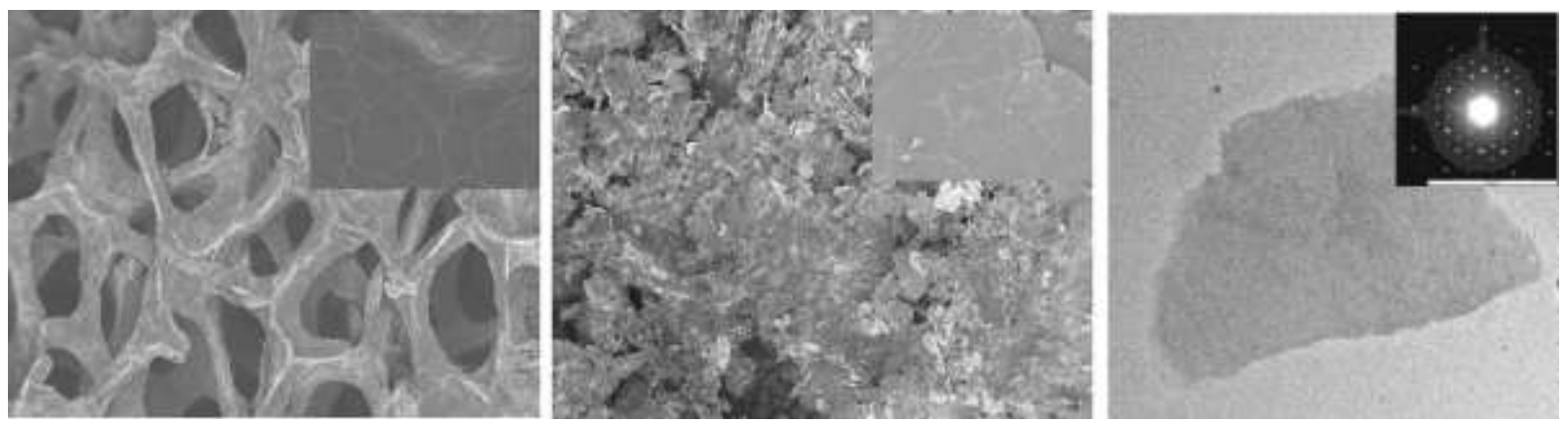

Figure 5 SEM images of (a) GF, (b) f-GF (c) TEM image of f-GF.

The electrochemical behavior of GF and f-GF was characterized by cyclic voltammetry (CV), galvanostatic charge-discharge (CD) and electrochemical impedance spectroscopy (EIS). Figure 6a compares CV curves for both GF and f-GF devices at a scan rate of $100 \mathrm{mV} \mathrm{s}^{-1}$. Both $\mathrm{CV}$ curves exhibit rectangular shape which demonstrates pure electrical double-layer capacitors (EDLC) behaviour. Inset show the CV of the GF, however as can be seen in main figure that the f-GF device exhibits a superior current response when compared to GF 

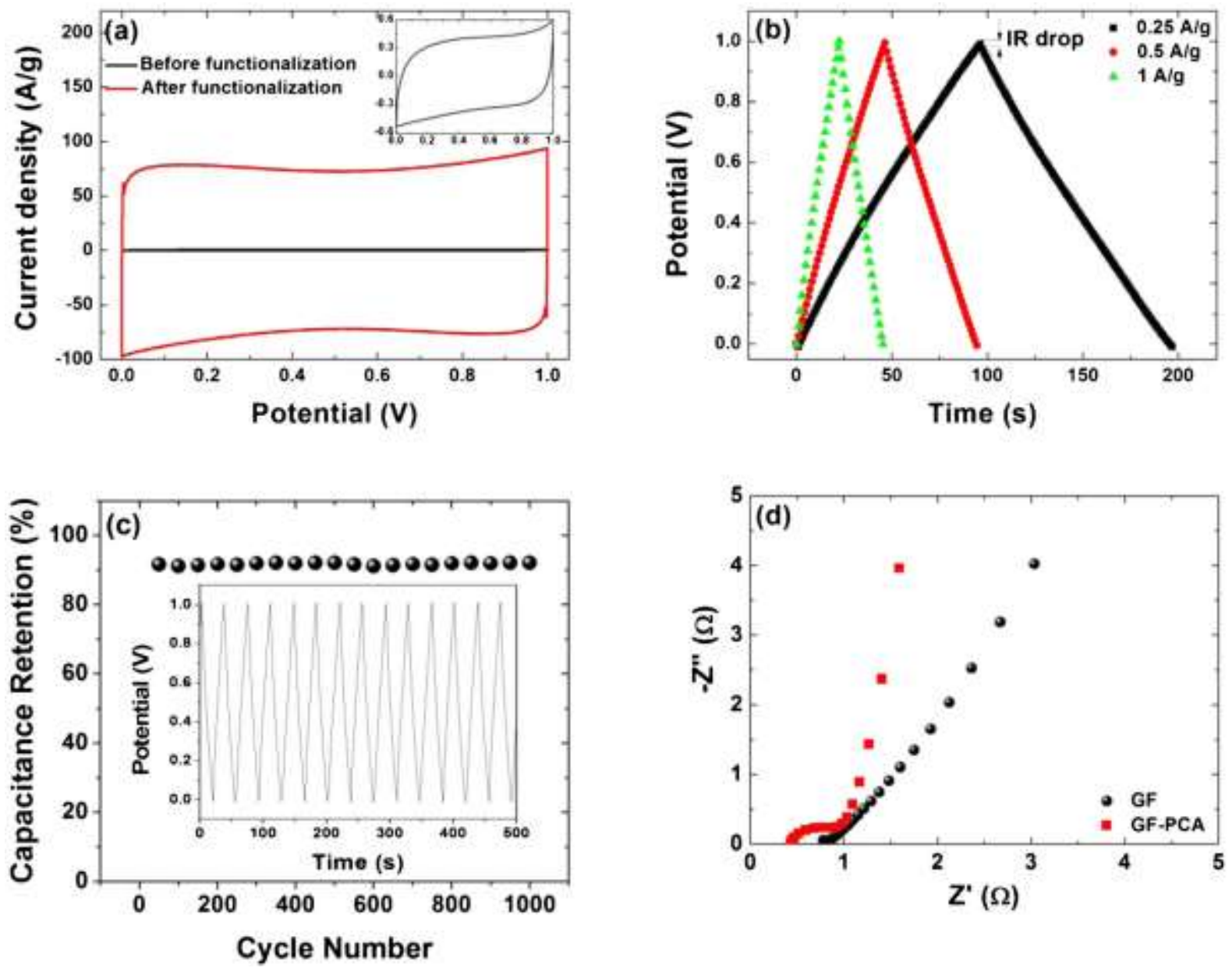

Figure 6 (a) $\mathrm{CVs}$ of GF and f-GF at scan rate of $100 \mathrm{mV} \mathrm{s}^{-1}$, (b) galvanostatic charge-discharge curves at three different current densities of $\mathrm{f}-\mathrm{GF}$, (c) the capacity retention of the composite at a current density of $0.5 \mathrm{~A} \mathrm{~g}^{-1}$, inset shows the continuous charge-discharge curve, (d) Nyquist plot for the GF and f-GF.

device. This could be attributed to the presence of oxygen containing functionalities contributing to the EDLC of the GF.

Cyclic performance and high life-cycle stability of an electrode material are very important parameters for evaluating a supercapacitor device for practical applications. Galvanostatic charge-discharge (CD) measurements were performed on the device at different current densities in the potential range of 0 and $1 \mathrm{~V}$ and are shown in figure $6 \mathrm{~b}$. It is observed that the $\mathrm{CD}$ curves were linear and symmetrical which are characteristic of ideal capacitor behaviour. The voltage (IR) drop observed (from charge discharge curve) was also very negligible, which indicates low internal resistance for the supercapacitor device. The cell capacitance $C$ in $\mathrm{F}$ was then calculated from the galvanostatic charge-discharge curves according to equation 1 below: 


$$
C_{\text {cell }}=\frac{i \Delta t}{\Delta v}
$$

where $i$ is the constant current for charge-discharge, $\Delta t$ is the discharge time and $\Delta v$ is the discharge voltage.

The specific capacitance ( $C_{\mathrm{sp}}$ in $\mathrm{F} \mathrm{g}^{-1}$ ) was then calculated according to equation 2 below:

$$
C_{\mathrm{sp}}=\frac{4 C_{\mathrm{cell}}}{m}
$$

where $m$ is the mass of active material in a single electrode in the cell.

The capacitance of the cell was calculated using equation (1) while the maximum specific capacitance of the electrode material obtained using equations (2) was $133.3 \mathrm{~F} \mathrm{~g}^{-1}$ at a current density of $0.25 \mathrm{~A} \mathrm{~g}^{-1}$. In comparison with device made from the GF, the specific capacitance and energy density were found to be orders of magnitude smaller in values as shown in our previous work [48].

The energy storage (E) per unit mass of the f-GF electrode and the maximum energy density of the coin cell were calculated using the equation 3 below for $C$ corresponding to $C_{\mathrm{sp}}$ and $C_{\text {cell }}$ respectively. The values were found to be $18.5 \mathrm{~W} \mathrm{~h} \mathrm{~kg}^{-1}$ and $4.7 \mathrm{~W} \mathrm{~h} \mathrm{~kg}^{-1}$, while the maximum power density $\left(P_{\max }\right)$ for the f-GF device was calculated using equation 4 below and was found to be $145.3 \mathrm{~kW} \mathrm{~kg}^{-1}$.

$$
\begin{aligned}
& E=\frac{1}{2} \frac{C V^{2}}{M}, \\
& P_{\max }=\frac{V^{2}}{4 M R_{\mathrm{s}}}
\end{aligned}
$$

Where $V$ is voltage applied, $R_{\mathrm{S}}$ is the equivalent series resistance (ESR) obtained from the intercept of the real axis from the Nyquist and $M$ is the total mass of active electrode material in the cell.

The long-term cycling stability of supercapacitor is also a significant parameter for their practical application. Figure $6 \mathrm{c}$ shows the variation of capacitance retention of $\mathrm{f}-\mathrm{GF}$ with cycle number for a potential between 0 and $1 \mathrm{~V}$. It is obvious that f-GF based device displays excellent cycling stability over the entire cycle numbers and 
retains $91 \%$ of the initial capacitance. This result indicates that f-GF based supercapacitor has good cycling stability and high reversibility in the repetitive charge-discharge process.

Electrochemical impedance spectroscopy (Nyquist plot) is a powerful tool for analysis of the electrochemical characteristics of electrode/electrolyte interfacial processes and evaluation of rate constants. Fig. 6d shows EIS of both GF and the f-GF electrodes. It is a representation of the real and imaginary parts of the impedance of the device. The plot is divided into two regions; a partial semicircle in the high-frequency region, which is characteristic of the charge transfer process taking place at the electrodelelectrolyte interface and a straight line in the low-frequency region, which represents the electron-transfer diffusion process [49]. The solution resistance refers to the resistance from the electrolyte, and the charge-transfer resistance corresponds to the total resistance at the electrodelelectrolyte interface. The intercept at the high frequency region on the $\mathrm{x}$-axis corresponds to the internal resistance of the electrolyte/electrode and is also referred to as the equivalent series resistance (ESR) which includes the resistance of aqueous electrolyte, the intrinsic resistance of the f-GF material and the contact resistance at the electrode interface. From the figure, the ESR value for the electrodes of the f-GF was found to be $0.43 \Omega$. which is smaller than that of GF $(0.9 \Omega)$. This value demonstrates that there is efficient exchange of ions at the electrode/electrolyte interface.

\section{Conclusions}

In summary, based on the results obtained it shows that PCA can be used successfully to functionalize GF without disrupting its $\mathrm{sp}^{2}$ hybrid structure via non covalent functionalization approach. This technique not only ensures efficient usage of the surface area offered by the foam but also reduces the agglomeration of the sheets due to the presence of oxygen containing groups, and hydroxyls from the PCA molecules which lead to improve capacitance of the GF. The results clearly show that electrochemical performance of GF can be improved via functionalization with electro active materials.

\section{Acknowledgements}

This work is based upon research supported by the South African Research Chairs Initiative of the Department of Science and Technology (SARCHi-DST) and the National Research Foundation (NRF). Any opinion, findings and conclusions or recommendations expressed in this work are those of authors and therefore the NRF and DST do not accept any liability with regard thereto. A. Bello, D. Y. Momodu and M. Fabiane acknowledge financial support from University of Pretoria and NRF for PhD bursaries. 


\section{References}

1. Geim A, Novoselov KS (2007) The rise of graphene. Nat Mater 6:183-191

2. Balandin A, Ghosh S, Bao W (2008) Superior thermal conductivity of single-layer graphene. Nano Lett 8:907-907

3. Novoselov KS, Geim A, Morozov SV, Jiang D, Zhang Y, Dubonos SV, Grigorieva IV, Firsov AA (2004) Electric field effect in atomically thin carbon films. Science 306:666-669

4. Lee C, Wei X, Kysar JW, Hone J (2008) Measurement of the Elastic Properties and Intrinsic Strength of Monolayer Graphene. Science 321: 385-388

5. Zhu Y, James DK, Tour JM (2012) New routes to graphene, graphene oxide and their related applications. Adv Mater 24:4924-4955

6. Chen D, Feng H, Li J (2012) Graphene oxide: preparation, functionalization, and electrochemical applications. Chem Rev 112:6027-6053

7. Zhu Y, Murali S, Cai W, Li X Suk JW, Potts JR, Ruoff RS (2010) Graphene and graphene oxide: synthesis, properties, and applications. Adv Mater 22:3906-3924

8. Dua V, Surwade SP, Ammu S, Agnihotra SR, Jain S, Roberts KE, Park S, Ruoff RS, Manohar SK (2010) All-organic vapor sensor using inkjet-printed reduced graphene oxide. Angew Chem Int Ed 49:2154-7

9. Eda G, Unalan HE, Rupesinghe N, Amaratunga GAJ, Chhowalla M (2008) Field emission from graphene based composite thin films. Appl Phys Lett 93:233502-1-233502-3

10. Dimitrakakis GK, Tylianakis E, Froudakis GE (2008) Pillared graphene: a new 3-D network nanostructure for enhanced hydrogen storage. Nano Lett 8:3166-3170

11. Conway BE (1999) Electrochemical Supercapacitors Scientific Fundamentals and Technological Applications

12. Hall PJ, Mirzaeian M, Fletcher SI, Sillars FB, Rennie AJR, Shitta-Bey GO, Wilson G, Cruden A,Carter R (2010) Energy storage in electrochemical capacitors: designing functional materials to improve performance. Energy Environ Sci 3:1238-1251

13. Wang Y, Cheng L, Xia Y-Y (2006) Electrochemical profile of nano-particle CoAl double hydroxide/active carbon supercapacitor using KOH electrolyte solution. J Power Sources 153:191-196

14. Yan J, Wei T, Shao B, Ma F, Fan Z, Zhang M, Zheng C, Shang Y, Qian W, Wei F (2010) Electrochemical properties of graphene nanosheet/carbon black composites as electrodes for supercapacitors. Carbon 48:1731-1737

15. Frackowiak E, Beguin F, (2001) Carbon materials for the electrochemical storage of energy in capacitors. Carbon 39:937-950

16. Du C, Pan N (2006) High power density supercapacitor electrodes of carbon nanotube films by electrophoretic deposition. Nanotechnology 17:5314-5318

17. Liu C, Yu Z, Neff D, Zhamu A, Jang BZ (2010) Graphene-Based Supercapacitor with an Ultrahigh Energy Density. Nano Lett 10:4863-4868 
18. Hummers W, Offeman R (1958) Preparation of graphitic oxide. J Am Chem Soc 80:1339-1339

19. Stankovich S, Dikin DA, Piner RD, Kohlhaas KA, Kleinhammes A, Jia Y, Wu Y, Nguyen ST, Ruoff RS (2007) Synthesis of graphene-based nanosheets via chemical reduction of exfoliated graphite oxide. Carbon 45:1558-1565

20. Park S, An J, Jung I, Piner RD, An SJ, Li X, Velamakanni V, Ruoff RS (2009) Colloidal suspensions of highly reduced graphene oxide in a wide variety of organic solvents. Nano Lett 9:1593-1597

21. Loh KP, Bao Q, Ang PK, Yang J (2010) The chemistry of graphene. J Mater Chem 2277-2289

22. Dreyer DR, Park S, Bielawski CW, Ruoff RS (2010) The chemistry of graphene oxide. Chem Soc Rev 39:228-240

23. Fang Y, Luo B, Jia Y, Li X, Wang B, Song Q, Kang2 F, Zhi L (2012) Renewing functionalized graphene as electrodes for high-performance supercapacitors. Adv Mater 24:6348-55

24. Su Q, Pang S, Alijani V, Li C, Feng X and Mullen K (2009) Composites of graphene with large aromatic molecules. Adv Mater 21:3191-3195

25. Niyogi S, Bekyarova E, Itkis ME, McWilliams JL, Hamon MA, Haddon RC (2006) Solution properties of graphite and graphene. J Am Chem Soc 128:7720-7721

26. Park S, Dikin DA, Nguyen ST, Ruoff RS (2009) Graphene oxide sheets chemically cross-linked by polyallylamine. J Phys Chem C 113:15801-15804

27. Salavagione HJ, Gómez MA, Martínez G (2009) Polymeric modification of graphene through esterification of graphite oxide and poly (vinyl alcohol). Macromolecules 42:6331-6334

28. Hou S, Kasner ML, Su S, Patel K and Cuellari R (2010) Highly sensitive and selective dopamine biosensor fabricated with silanized graphene. J Phys Chem C 114:14915-14921

29. Yang H, Li F, Shan C, Han D, Zhang Q, Niu Lab, Ivaska A (2009) Covalent functionalization of chemically converted graphene sheets via silane and its reinforcement. J Mater Chem 19:4632

30. Liu H, Liu Y, Zhu D (2011) Chemical doping of graphene. J Mater Chem 21:3335

31. Xu Y, Bai H, Lu G, et al. (2008) Flexible Graphene Films via the Filtration of Water-Soluble Noncovalent Functionalized Graphene Sheets. J Am Chem Soc 130:5856-5857

32. Dong X, Fu D, Fang W, Li C, Shi G (2009) Doping Single Layer Graphene with Aromatic Molecules. Small 5:1422-1426

33. Dong X, Shi Y, Zhao Y, Chen D, Ye J, Yao Y, Gao F, Ni Z, Yu T, Shen Z, Huang Y, Chen P, Li L-J, (2009) Symmetry breaking of graphene monolayers by molecular decoration. Phys Rev Lett 102:135501135504

34. Strobel P, Riedel M, Ristein J, Ley L (2004) Surface transfer doping of diamond. Nature 430:439-441

35. Hsu C-L, Lin C-T, Huang J-H, Chu C-W, Wei K-H, Li L-J (2012) Layer-by-layer graphene/TCNQ stacked films as conducting anodes for organic solar cells. ACS Nano 6:5031-5039

36. Liu H, Gao J, Xue M, Zhu N, X Zhang M, Cao T. (2009) Processing of graphene for electrochemical application: noncovalently functionalize graphene sheets with water-soluble electroactive methylene green. Langmuir 25:12006-12010. 
37. Shang NG, Papakonstantinou P, McMullan M, Chu M, Stamboulis A, Potenza A, Dhesi SS, Marchetto H (2008) Catalyst-Free Efficient Growth, Orientation and Biosensing Properties of Multilayer Graphene Nanoflake Films with Sharp Edge Planes. Adv Funct Mater 18:3506-3514

38. Ghosh S, An X, Shah R, Rawat D, Dave B,S Kar S, Talapatra S (2012) Effect of 1- Pyrene CarboxylicAcid Functionalization of Graphene on Its Capacitive Energy Storage. J Phys Chem C 116:20688-20693

39. An X, Simmons T, Shah R, Wolfe C, Lewis KM, Washington M, Nayak SK, Talapatra S, Kar S (2010) Stable aqueous dispersions of noncovalently functionalized graphene from graphite and their multifunctional high-performance applications. Nano Lett 10:4295-301

40. Chen Z, Ren W, Gao L, Liu B, Pei S, Cheng H-M (2011) Three-dimensional flexible and conductive interconnected graphene networks grown by chemical vapour deposition. Nat Mater 10:424-428

41. Bello A, Makgopa K, Fabiane M, Dodoo-Ahrin D, Ozoemena KI, Manyala N (2013) Chemical adsorption of $\mathrm{NiO}$ nanostructures on nickel foam-graphene for supercapacitor applications. J Mater Sci (2013) 48:6707-6712

42. Ferrari A (2007) Raman spectroscopy of graphene and graphite: disorder, electron-phonon coupling, doping and nonadiabatic effects. Solid State Commun 143:47-57

43. Kitaura R, Imazu N, Kobayashi K, Shinohara H (2008) Fabrication of metal nanowires in carbon nanotubes via versatile nano-template reaction. Nano Lett 8:693-699

44. Zhou J, Song H, Ma L, Chen X (2011) Magnetite/graphene nanosheet composites: interfacial interaction and its impact on the durable high-rate performance in lithium-ion batteries. RSC Adv 1:782-791

45. Hu J, Ramadan A, Luo F, Qi B, Deng X, Chen J (2011) One-step molybdate ion assisted electrochemical synthesis of $\alpha-\mathrm{MoO}_{3}$-decorated graphene sheets and its potential applications. J Mater Chem 21:1500915014

46. Park S, An J, Piner RD, Jung I, Yang D, Velamakanni A, Nguyen ST, Ruoff RS(2008) Aqueous suspension and characterization of chemically modified graphene sheets. Chem Mater 20:6592-6594

47. Chae SJ, Gunes F, Kim KK, Kim ES, Han GH, Kim SM, Shin H-J, Yoon S-M, Choi J-Y, Park MH, Yang CW, Pribat D, Lee YH (2009) Synthesis of Large-Area Graphene Layers on Poly-Nickel Substrate by Chemical Vapor Deposition: Wrinkle Formation. Adv Mater 21:2328-2333

48. Bello A, Fashedemi OO, Lekitima JN, Fabiane M, Dodoo-Arhin D, Ozoemena KI, Gogotsi Y, Johnson ATC, Manyala N (2013) High-performance symmetric electrochemical capacitor based on graphene foam and nanostructured manganese oxide. AIP Adv 3:82118

49. Gamby J, Taberna PL, Simon P, Fauvarque JF, Chesneau M. (2001) Studies and characterisations of various activated carbons used for carbon/carbon supercapacitors. J Power Sources 101:109-116 